\title{
Modeling the effects of the inlet manifold design on the performance of a diesel oxidation catalytic converter
}

Sruti Dammalapati, Preeti Aghalayam†, Niket Kaisare*

Department of Chemical Engineering, Indian Institute of Technology Madras, Chennai 600036, India

†Email: preeti@iitm.ac.in; Phone: [+91] (44) 22574185

*Email: nkaisare@iitm.ac.in; Phone: [+91] (44) 22574176

\section{Supporting Information}

\section{S.1. GRID INDEPENDENCE}

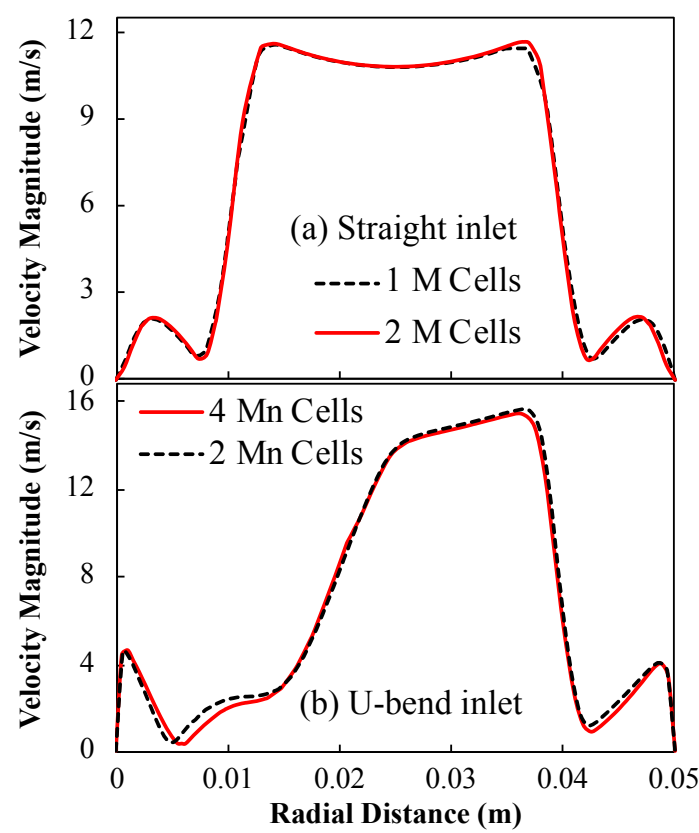

Figure S1: Grid independence study showing the effect of the number of cells on the velocity magnitude predicted upstream of the monolith in the catalytic converter with (a) straight and

(b) U-bend inlet manifold geometry.

Prior to using the models, grid independence was carefully verified for both cold-flow as well as non-isothermal simulations of the full geometry with DOC reactions included. Figure S1 shows the result of grid independence study, where the velocity magnitude along the radial 
direction at a location upstream of the monolith is plotted. We verified both structured and unstructured grids and found that structured grids performed better than the unstructured ones, especially with the DOC reactions included. A superfine mesh with 1 million was used for the straight inlet manifold and the CFD predictions were compared with those obtained by doubling the grids. As seen from Figure S1(a), doubling the mesh further did not affect the results significantly. A similar study for the u-bend inlet manifold geometry, presented in Figure S1(b), shows that the grid with 2 million and 4 million cells showed quantitatively close results.

\section{S.2. BENCHMARKING DOC KINETICS IN FLUENT}

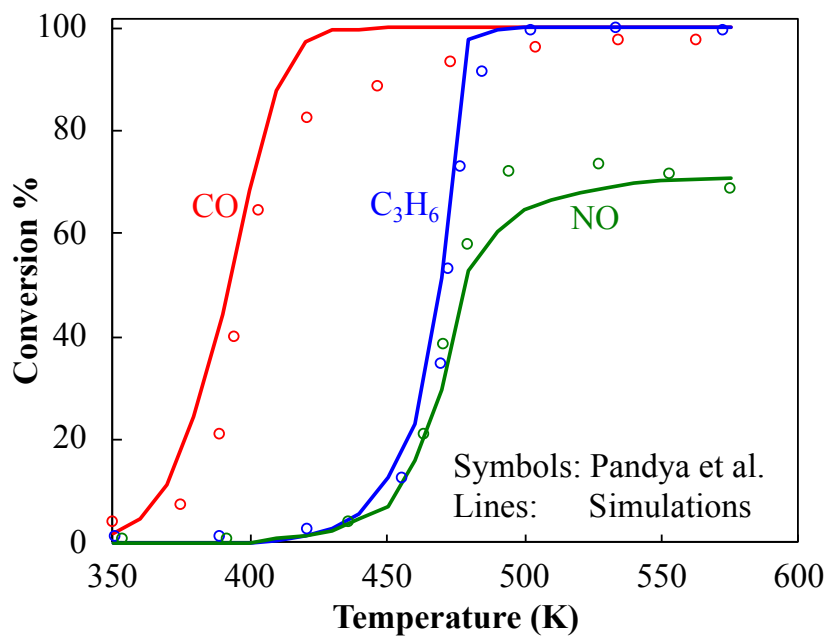

Figure S2: A comparison of model predictions for DOC simulations (lines) in our work and the experimental data from Pandya et al. (2009)

Finally, Figure $\mathrm{S} 2$ shows the variation in $\mathrm{CO}, \mathrm{HC}$ and $\mathrm{NO}$ conversion vs. inlet temperature for the DOC reactions included in the CFD model and the reactor geometry based on the work of Pandya et al. (2009). The lines denote our simulation results and symbols represent their experimental data. A reasonable match between our simulations and experimental data was obtained.

\section{S.3. UDF FOR DOC KINETICS}

The DOC kinetic model was implemented in Fluent using the DEFINE_SR_RATE macro which is a user defined function in C. The UDF is provided below: 
Custom surface reaction rate UDF

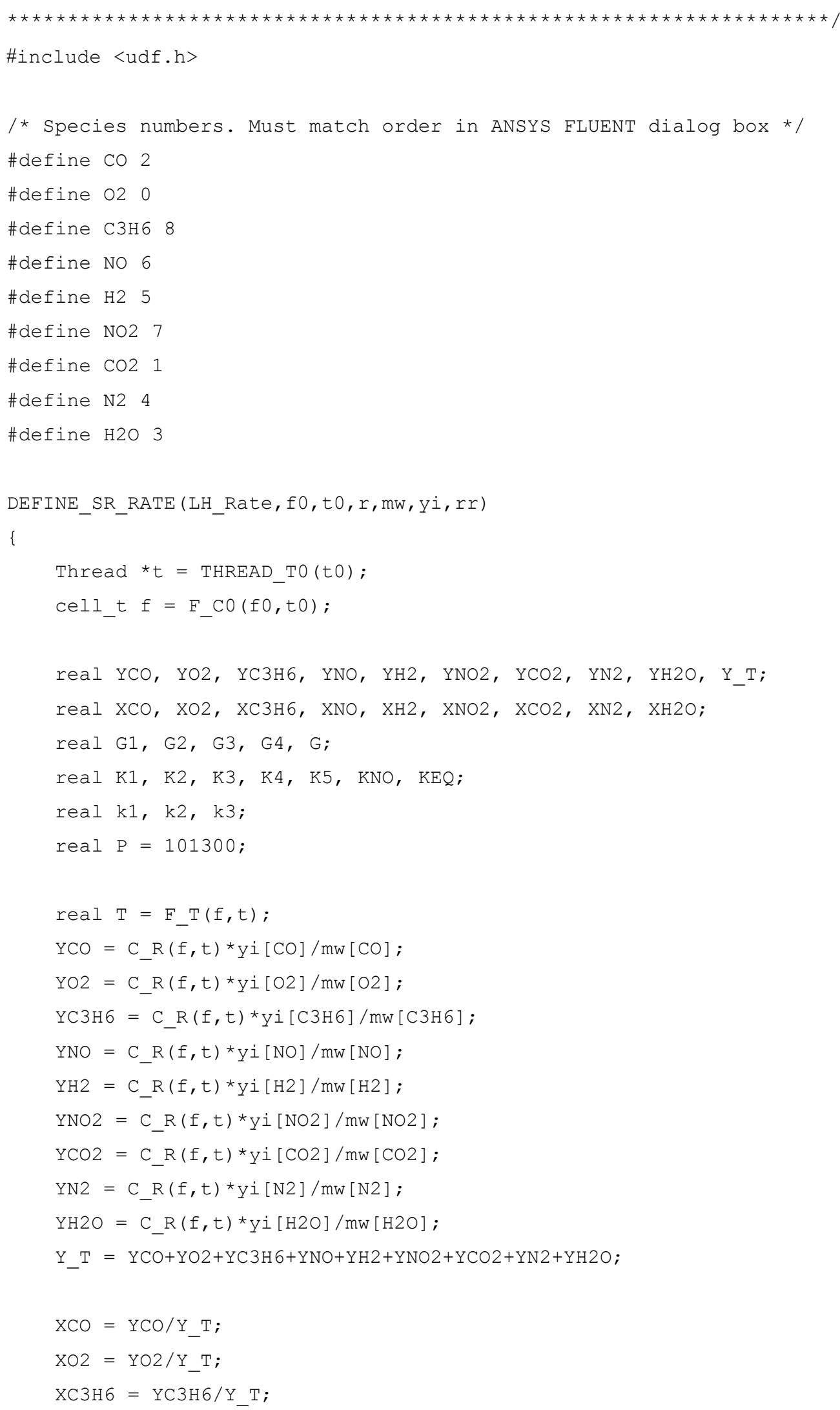




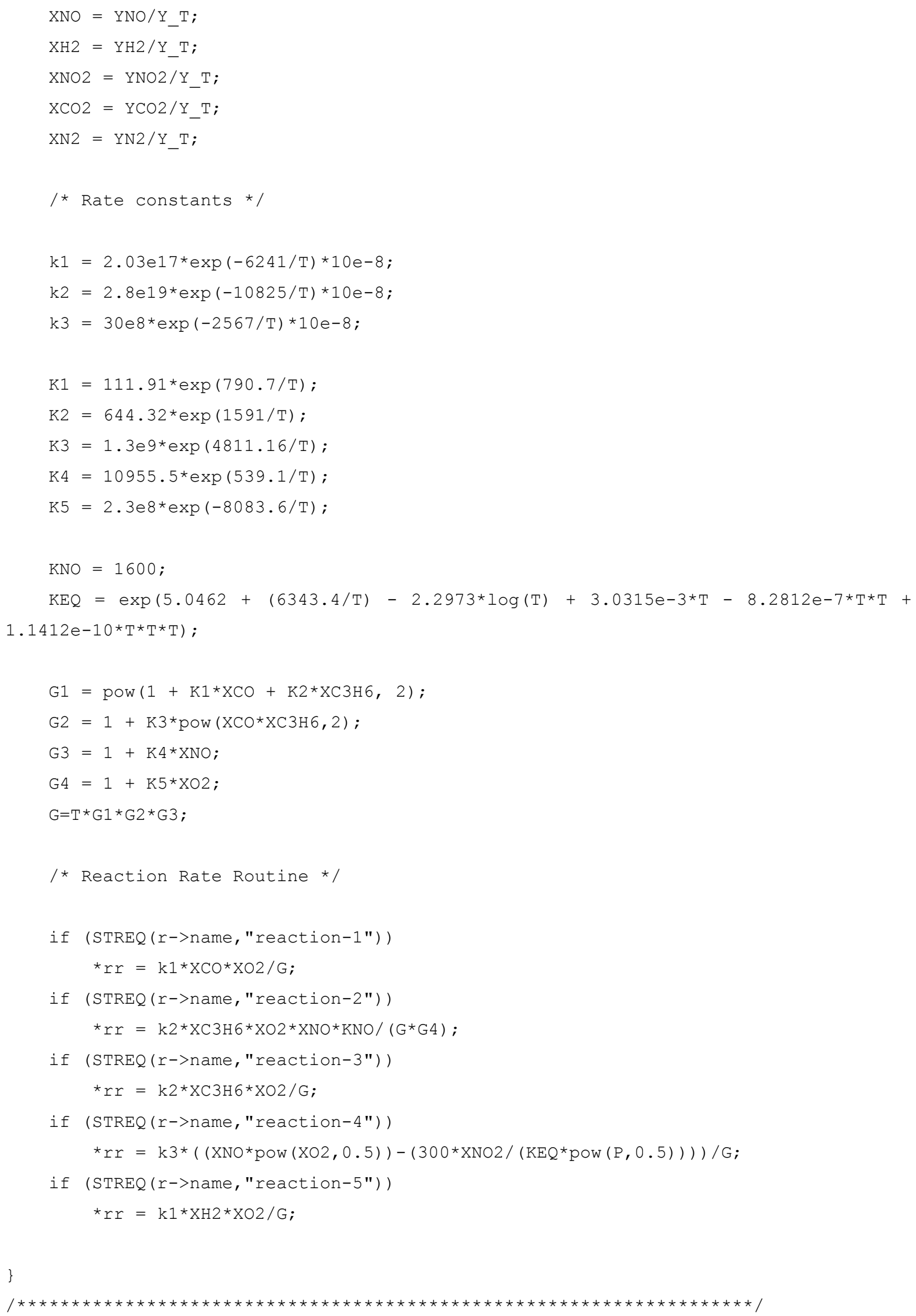




\section{S.4. BALANCE EQUATIONS}

The balance equations used in ANSYS Fluent for momentum, species, energy conservation and turbulence model implemented are summarized in Table S1.

Table S1: Balance equations used in ANSYS Fluent

\section{Description Balance Equation}

Momentum

conservation

$\frac{\partial(\rho \vec{v})}{\partial t}+\nabla \cdot(\rho \vec{v} \vec{v})=-\nabla p+\nabla \cdot(\overline{\bar{\tau}})+\rho \vec{g}+\vec{F}$

$p$ is the static pressure, $\rho$ is the density, $\vec{v}$ is the velocity field, $\overline{\bar{\tau}}$ is the stress tensor, $\rho \vec{g}$ is the the gravitational body force and $\vec{F}$ is the source term for porous media.

$\vec{F}=S_{i}=-\left(\sum_{j=1}^{3} \alpha_{i j} \mu v_{j}+\sum_{j=1}^{3} \beta_{i j} \frac{1}{2} \rho|v| v_{j}\right)$

$\alpha_{i j}$ and $\beta_{i j}$ are diagonal matrices representing the viscous and inertial resistance coefficients, respectively. In the direction of flow, $\alpha_{i}=$ $3.846 \times 10^{7} \mathrm{~m}^{-2}$ and $\beta_{i}=20.414 \mathrm{~m}^{-1}$

$\overline{\bar{\tau}}=\mu\left[\left(\nabla \vec{v}+\nabla \vec{v}^{T}\right)-\frac{2}{3} \nabla \cdot \vec{v} I\right.$

$\mu$ is the molecular viscosity, $I$ is the unit tensor, and the second term on the right hand side is the effect of volume dilation.

Reaction-diffusion balance for surface $\rho_{\text {wall }} D_{i} \frac{\partial Y_{i, \text { wall }}}{\partial n}-\dot{m}_{\text {dep }} Y_{i, \text { wall }}=M_{w, i} \hat{R}_{i, \text { gas }}$ chemistry $\frac{\partial\left[S_{i}\right]_{\text {wall }}}{\partial t}=\hat{R}_{i, \text { site }}$

The mass flux of each gas species due to diffusion and convection to/from the surface is balanced with its rate of consumption/production on the reacting surface. $\dot{m}_{d e p}$ is the net rate of mass deposition due to surface reaction and the wall mass fraction $Y_{i, \text { wall }}$ is related to concentration $\left[G_{i}\right]_{\text {wall }}$

$$
\left[G_{i}\right]_{\text {wall }}=\frac{\rho_{\text {wall }} Y_{i, \text { wall }}}{M_{w, i}}
$$



Turbulence model $\frac{\partial(\rho \omega)}{\partial t}+\frac{\partial\left(\rho \omega u_{i}\right)}{\partial x_{i}}=\frac{\partial}{\partial x_{j}}\left(\Gamma_{\omega} \frac{\partial \omega}{\partial x_{j}}\right)+G_{\omega}-Y_{\omega}$
$\frac{\partial(\rho k)}{\partial t}+\frac{\partial\left(\rho k u_{i}\right)}{\partial x_{i}}=\frac{\partial}{\partial x_{j}}\left(\Gamma_{k} \frac{\partial k}{\partial x_{j}}\right)+G_{k}-Y_{k}$
$G_{k}$ represents the generation of turbulence kinetic energy and $G_{\omega}$ represents the generation of $\omega . \Gamma_{k}$ and $\Gamma_{\omega}$ represent the effective diffusivity of $k$ and $\omega$, respectively. $Y_{\omega}$ and $Y_{k}$ represent the dissipation of $k$ and $\omega$ due to turbulence.

Energy

conservation

$\frac{\partial(\rho \mathrm{E})}{\partial t}+\nabla \cdot(\vec{v}(\rho \mathrm{E}+\mathrm{p}))=-\nabla \cdot\left(k_{e f f} \nabla T-\sum_{j} h_{j} \vec{J}_{j}+\left(\overline{\bar{\tau}}_{e f f} \cdot \vec{v}\right)\right)+S_{h}$

$\mathrm{E}=\mathrm{h}-\frac{p}{\rho}+\frac{v^{2}}{2}$

$k_{e f f}$ is the effective thermal conductivity, $\overrightarrow{J_{i}}$ is the diffusive flux of species $j$. The first three terms on the right-hand side represent energy transfer due to conduction, species diffusion, and viscous dissipation, respectively. $S_{h}$ includes the heat of chemical reaction.

In solid regions, the energy transport equation used is

$\frac{\partial(\rho \mathrm{h})}{\partial t}+\nabla \cdot(\vec{v} \rho \mathrm{h})=-\nabla \cdot(k \nabla T)$

Where $\mathrm{h}$ is the ensible enthalpy and $k$ is the conductivity. 